Pacific Journal of Mathematics

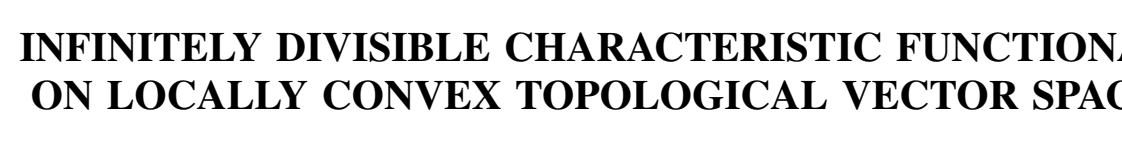




\title{
INFINITELY DIVISIBLE CHARACTERISTIC FUNCTIONALS ON LOCALLY CONVEX TOPOLOGICAL VECTOR SPACES
}

\author{
B. L. S. Prakasa RaO
}

\begin{abstract}
S. Johansen has given a characterization of infinitely divisible characteristic functions on the real line analogous to the Bochner's theorem characterizing the characteristic functions through their nonnegative definiteness. Recently the present author was able to extend this result to infinitely divisible characteristic functionals on a Hilbert space and on locally compact abelian groups. We shall now obtain a similar theorem for infinitely divisible characteristic functionals on locally convex topological vector spaces whose dual spaces are nuclear.
\end{abstract}

Let $E$ be a locally convex separable topological vector space. Let $U$ be an open circled convex neighborhood of zero. For every $x \in E$, let

$$
\|x\|_{U}=\operatorname{Inf}\{\lambda: \lambda>0, x \in \lambda U\} .
$$

$\|x\|_{U}$ is a semi-norm on $E$. Let $E_{U}$ denote the associated norm space (quotient space of $E$ by the subspace such that $\|x\|_{U}=0$ ). Its completion is the Banach space $\hat{E}_{U}$. If $\hat{E}_{U}$ is a Hilbert space, then $U$ is called a prehilbertian neighborhood. Let $\pi_{U V}$ denote the canonical mapping from $E_{U}$ to $E_{V}$ extended by continuity from $\hat{E}_{U}$ to $\hat{E}_{V}$. A nuclear space is a separated locally convex space such that there exists a fundamental system $\mathscr{T}$ of neighborhoods of zero which are prehilbertian neighborhoods and such that for every $U \in \mathscr{T}$, there exists a $V \in \mathscr{T}, V \subset U$ such that the mapping from $\hat{E}_{U}$ to $\hat{E}_{V}$ is Hilbert-Schmidt. Let $\mathscr{B}$ denote the $\sigma$-algebra of Borel subsets of $E$ i.e., the $\sigma$-algebra generated by the open sets in $E$. A probability measure $\mu$ on the measurable space $(E, \mathscr{B})$ is a measure $\mu$ such that $\mu(E)=1$. A probability measure $\mu$ is said to be tight if given any $\varepsilon>0$, there exists a compact set $K_{\varepsilon}$ such that $\mu\left(K_{\varepsilon}\right)>1-\varepsilon$. Let $F$ denote the dual space of $E$. The characteristic functional $\varphi(y)$ for $y \in F$ is defined by the relation

$$
\varphi(y)=\int_{E} e^{i<x, y>} \mu(d x) .
$$

It can be shown that this functional $\varphi$ possess the following properties. (1) $\varphi(0)=1$. (2) $\varphi$ is positive-definite, i.e., for any $N$, any $y_{1}, y_{2}, \cdots, y_{N} \in F$ and any complex numbers $\alpha_{1}, \cdots, \alpha_{N}$, 


$$
\sum_{i=1}^{N} \sum_{j=1}^{N} \varphi\left(y_{i}-y_{j}\right) \alpha_{i} \bar{\alpha}_{j} \geqq 0 .
$$

(3) For every fixed $y \in F$, the function $\varphi(t y)$ of a real argument $t$ is continuous. In general, these three properties do not characterize the characteristic functionals of countably additive probability measures $\mu$ on $(E, \mathscr{B})$. Any functional $\varphi$ on $F$ which satisfies the properties (1), (2), and (3) is the characteristic functional of cylinder set measure or a weak distribution on $E$. For the definitions of cylinder set measures or weak distributions and other results, the reader is referred to Prohorov [9], Mourier [6] or Gelfand and Vilenkin [3].

The following theorem can be proved from the results of Minlos [5] and Schwartz [10] as pointed out by Mourier [6]. Recently Badrikian [1] was able to extend this results to more general spaces.

Minlos-Schwartz Theorem. Let $(E, \mathscr{T})$ be a locally convex separable topological vector space such that the dual space $F$ is nuclear when provided with the topology of uniform convergence on compact convex subsets of $E$. Then every positive definite continuous functional $\varphi$ on $F$ with $\varphi(0)=1$ is the characteristic functional of a tight probability measure on $(E, \mathscr{B})$.

Our aim in this paper is to give a similar characterization for infinitely divisible characteristic functionals on locally convex separable topological vector spaces $E$ whose dual spaces $F$ are nuclear when provided with the topology of uniform convergence on compact convex subsets of $E$. Similar results were obtained by Johansen [4] in the case of real line and by Prakasa Rao $[7,8]$ for Hilbert spaces and locally compact abelian groups.

2. Infinitely divisible distributions. Unless otherwise stated, we shall suppose here after that $E$ is a locally convex separable topological vector space, $\mathscr{B}$ is the $\sigma$-field of Borel subsets of $E$ and the dual space $F$ of $E$ is nuclear when provided with the topology of uniform convergence on compact convex subsets of $E$. A tight probability measure $\mu$ on $(E, \mathscr{B})$ is said to be infinitely divisible if there exists another probability measure $\lambda_{n}$ on $(E, \mathscr{B})$ such that $\mu=\lambda_{n}^{n}$ for each $n$ where $\lambda_{n}^{n}$ denotes the measure obtained by convoluting $\lambda_{n}$ for $n$ times. Structures of tight infinitely divisible laws and Levy-Khintchin type representation of their characteristic functionals have been discussed by Tortrat [11, 12] and Fernique [2]. It is clear from the theorem and the corollary in Tortrat [12] (see page 325) that the characteristic functional $\varphi($.$) of a tight infinitely$ divisible law on $(E, \mathscr{B})$ does not vanish and $\varphi(y)=\exp \psi(y)$ where 
$\psi($.$) is a complex-valued function on F$ with $\psi(0)=0$. We shall call $\psi$ the logarithm of the characteristic functional $\varphi$.

MaIN Theorem. Let $(E, \mathscr{T})$ be a locally convex separable topological vector space such that the dual space $F$ is nuclear when provided with the topology of uniform convergence on compact convex subsets of $E$. Then a necessary and sufficient condition for a complex-valued functional $\psi$ on $F$ to be the logarithm of the characteristic functional of a tight infinitely divisible probability measure $\mu$ on $(E, \mathscr{B})$ is that

(i) $\psi(0)=0, \psi(y)=\overline{\psi(-y)}$ for $y \in F$,

(ii) for every choice $y_{i}, 1 \leqq i \leqq N$ in $F$ and complex numbers $\alpha_{i}, 1 \leqq i \leqq N$ such that $\sum_{i=1}^{N} \alpha_{i}=0$,

$$
\sum_{i=1}^{N} \sum_{j=1}^{N} \psi\left(y_{i}-y_{j}\right) \alpha_{i} \bar{\alpha}_{j} \geqq 0, \text { and }
$$

(iii) $\psi$ is a continuous functional on $F$.

We shall now state a lemma which will be used in the proof of the main theorem.

LEMMA. Let $\psi($.$) be any complex-valued functional on F$ such that $\psi(0)=0, \psi(y)=\overline{\psi(-y)}$. Then the following conditions are equivalent.

(1) For every choice $y_{i}, 1 \leqq i \leqq N$ in $F$ and complex numbers $\alpha_{i}, 1 \leqq i \leqq N$ and $\lambda>0$,

$$
\sum_{i=1}^{N} \sum_{j=1}^{N} e^{\lambda \psi\left(y_{i}-y_{j}\right)} \alpha_{i} \bar{\alpha}_{j} \geqq 0
$$

(2) For every choice $y_{i}, 1 \leqq i \leqq N$ in $F$ and complex numbers $\alpha_{i}, 1 \leqq i \leqq N$ such that $\sum_{i=1}^{N} \alpha_{i}=0$,

$$
\sum_{i=1}^{N} \sum_{j=1}^{N} \psi\left(y_{i}-y_{j}\right) \alpha_{i} \bar{\alpha}_{j} \geqq 0 \text {. }
$$

(3) For every choice $y_{i}, 1 \leqq i \leqq N$ in $F$ and complex numbers $\alpha_{i}, 1 \leqq i \leqq N$,

$$
\sum_{i=1}^{N} \sum_{j=1}^{N}\left[\psi\left(y_{i}-y_{j}\right)-\psi\left(y_{i}\right)-\psi\left(-y_{j}\right)\right] \alpha_{i} \bar{\alpha}_{j} \geqq 0 .
$$

This lemma has been proved for complex-valued functions on the real line by Johansen [4]. We shall omit the proof of this lemma as it is similar to the proof in the case of real line.

3. Proof of the main theorem. Suppose $\phi$ is the characteristic functional of a tight infinitely divisible probability measure $\mu$ on $(E, \mathscr{B})$. Let $\psi$ denote the logarithm of $\varphi$. (i) follows from the 
definition of the functional $\varphi$. Since $\mu$ is infinitely divisible, $\varphi^{1 / n}$ is a uniquely determined characteristic functional of probability measure for every $n$ and hence $\varphi^{r}$ is a characteristic functional of a probability measure for every rational number $r$. Hence by the property of a characteristic functional, for any choice of $y_{i}, 1 \leqq i \leqq N$ in $F$ and complex numbers $\alpha_{i}, 1 \leqq i \leqq N$, we have

$$
\sum_{i=1}^{N} \sum_{j=1}^{N}\left[\varphi\left(y_{i}-y_{j}\right)\right]^{r} \alpha_{i} \bar{\alpha}_{j} \geqq 0 \text {. }
$$

Let $r_{n}$ approach $\lambda>0$ as $n$ approaches infinity. Then it follows that

$$
\sum_{i=1}^{N} \sum_{j=1}^{N}\left[\varphi\left(y_{i}-y_{j}\right)\right]^{2} \alpha_{i} \bar{\alpha}_{j} \geqq 0
$$

which in turn proves that $\psi=\log \varphi$ satisfies the condition (ii) of the theorem in view of the lemma. Since $\varphi$ is a continuous functional on $F$ and is nonvanishing, $\psi=\log \varphi$ is a continuous functional on $F$. This proves the condition (iii) of the theorem which completes the proof of the necessity.

Conversely suppose $\psi$ is a complex-valued function satisfying conditions (i), (ii) and (iii) of the theorem. Let $\varphi(y)=\exp \psi(y)$ for $y \in F$. Clearly $[\varphi(0)]^{\lambda}=1$ for any $\lambda>0$ and $\varphi^{\lambda}$ is a continuous functional on $F$ for any $\lambda>0$. Since

$$
\sum_{i=1}^{N} \sum_{j=1}^{N} \psi\left(y_{i}-y_{j}\right) \alpha_{i} \bar{\alpha}_{j} \geqq 0
$$

for every choice of $y_{i}, 1 \leqq i \leqq N$ in $F$ and complex numbers $\alpha_{i}$, $1 \leqq i \leqq N$ such that $\sum_{i=1}^{N} \alpha_{i}=0$, it follows from the lemma,

$$
\sum_{i=1}^{N} \sum_{j=1}^{N} e^{\lambda \psi\left(y_{i}-y_{j}\right)} \alpha_{i} \bar{\alpha}_{j} \geqq 0
$$

or equivalently,

$$
\sum_{i=1}^{N} \sum_{j=1}^{N}\left[\varphi\left(y_{i}-y_{j}\right)\right]^{2} \alpha_{i} \bar{\alpha}_{j} \geqq 0
$$

for every choice of $y_{i}, 1 \leqq i \leqq N$ in $F$ and every choice of complex numbers $\alpha_{i}, 1 \leqq i \leqq N$. In other words $\phi^{\lambda}$ is a positive definite functional on $F$. Furthermore $\varphi^{\lambda}$ is a continuous functional on $F$ for any $\lambda>0$ since $\varphi$ is a continuous functional on $F$. Hence by the Minlos-Schwartz theorem, $\varphi^{\lambda}$ is a characteristic functional of a tight probability measure for every $\lambda>0$. In particular, it follows that $\phi^{1 / n}$ is a characteristic functional of a tight probability measure on $(E, \mathscr{B})$. Hence $\varphi$ is the characteristic functional of an infinitely divisible tight probability measure $\mu$ on $(E, \mathscr{B})$. This completes the proof of sufficiency.

4. Remarks. Similar results have been obtained for infinitely 
divisible characteristic functionals on locally compact abelian groups and on Hilbert spaces in Prakasa Rao $[7,8]$. Recently Badrikian [1] has extended Minlos-Schwartz theorem to more general spaces and our main theorem can also be extended to such spaces.

\section{REFERENCES}

1. A. Badrikian, Remarques sur les Theoremes de Bochner et P. Levy, Symposium on Probability methods in Analysis, Springer, Berlin- Heidelberg-New York, 1967.

2. X. Fernique, Lois indefiniment divisibles sur l' espace des distributions, Inventions Math. 3 (1967), 282-292.

3. I. M. Gelfand and N. Ya. Vilenkin, Generalized Functions, Vol. 4, Academic Press, New York, 1964.

4. S. Johansen, An application of extreme point methods to the representation of infinitely divisible distributions, Z. Wahrschrinlichkeitstheorie Verw. Geb. 5 (1966), 304-316.

5. R. A. Minlos, Generalized random processes and their extension in measure, Selected translations in Mathematical Statistics and Probability, Amer. Math. Soc. Providence, 1963, Vol. 3.

6. E. Mourier, Random elements in linear spaces, Proc. Fifth Berkeley Symp. Math. Statist. and Prob., Univ. of California Press, Berkeley and Los Angeles, 1967, Vol. 2, Part 1, 43-53.

7. B. L. S. Prakasa Rao, On a characterization of infinitely divisible characteristic functionals on a Hilbert space, Z. Wahrscheinlichkeitstheorie Verw. Geb. 14 (1970), 254-256.

8. - On a characterization of infinitely divisible characteristic functionals on locally compact abelian groups (submitted for publication)

9. Yu. V. Prohorov, The method of characteristic functionals, Proc. Fourth Berkeley Symp. Math. Statist. and Prob., Univ. of California Press, Berkeley and Los Angeles, 1960, Vol. 2, 403-419.

10. L. Schwartz, Measures de Radon sur des espaces topologiques arbitraires, Cours de 3d cycle 1964-1965, Institute Henri Poincare', Paris.

11. A. Tortrat, Structure des lois indefinimemt divisibles dans un espace Vectoriel topologique, Symposium on probability methods in analysis, Springer, Berlin-HeidelbergNew York, 1967.

12. - Sur la structure des lois indefiniment divisibles (classe $\mathscr{T}(X)$ ) dansles espaces vectoriels $X$ (sur le corps riel), Z. Wahrscheinlichkeitstheorie Verw. Geb. 11 (1969), 311-326.

Received November 13, 1969.

INDIAN INSTITUTE OF TECHNOLOGY

KANPUR, INDIA 



\section{PACIFIC JOURNAL OF MATHEMATICS}

\section{EDITORS}

H. SAmelson

Stanford University

Stanford, California 94305

RICHARd PieRCe

University of Washington

Seattle, Washington 98105

\author{
J. DugundJI \\ Department of Mathematics \\ University of Southern California \\ Los Angeles, California 90007 \\ RICHARD ARENS \\ University of California \\ Los Angeles, California 90024
}

ASSOCIATE EDITORS
E. F. BECKENBACH
B. H. NeumanN
F. WOLF
K. YosHIDA

\section{SUPPORTING INSTITUTIONS}

UNIVERSITY OF BRITISH COLUMBIA

CALIFORNIA INSTITUTE OF TECHNOLOGY

UNIVERSITY OF CALIFORNIA

MONTANA STATE UNIVERSITY

UNIVERSITY OF NEVADA

NEW MEXICO STATE UNIVERSITY

OREGON STATE UNIVERSITY

UNIVERSITY OF OREGON

OSAKA UNIVERSITY

UNIVERSITY OF SOUTHERN CALIFORNIA
STANFORD UNIVERSITY

UNIVERSITY OF TOKYO

UNIVERSITY OF UTAH

WASHINGTON STATE UNIVERSITY

UNIVERSITY OF WASHINGTON

AMERICAN MATHEMATICAL SOCIETY CHEVRON RESEARCH CORPORATION TRW SYSTEMS

NAVAL WEAPONS CENTER 


\section{Pacific Journal of Mathematics}

\section{Vol. 35, No. $1 \quad$ September, 1970}

B. D. Arendt and C. J. Stuth, On the structure of commutative periodic semigroups ..................................... 1

B. D. Arendt and C. J. Stuth, On partial homomorphisms of semigroups .... 7

Leonard Asimow, Extensions of continuous affine functions ............ 11

Claude Elias Billigheimer, Regular boundary problems for a five-term recurrence relation ................................... 23

Edwin Ogilvie Buchman and F. A. Valentine, A characterization of the

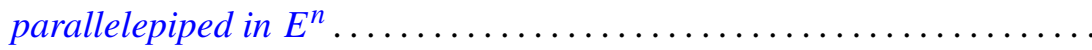
53

Victor P. Camillo, A note on commutative injective rings ............ 59

Larry Jean Cummings, Decomposable symmetric tensors ............. 65

J. E. H. Elliott, On matrices with a restricted number of diagonal values ... 79

Garth Ian Gaudry, Bad behavior and inclusion results for multipliers of type

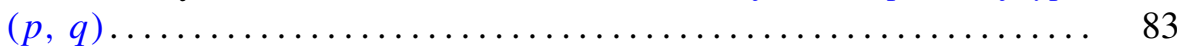

Frances F. Gulick, Derivations and actions .................. 95

Langdon Frank Harris, On subgroups of prime power index . . . . . . . . 117

Jutta Hausen, The hypo residuum of the automorphism group of an abelian

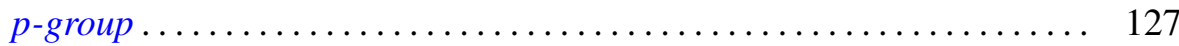

R. Hrycay, Noncontinuous multifuctions . ................... 141

A. Jeanne LaDuke, On a certain generalization of $p$ spaces ............ 155

Marion-Josephine Lim, Rank preservers of skew-symmetric matrices...... 169

John Hathway Lindsey, II, On a six dimensional projective representation of the Hall-Janko group ................................... 175

Roger McCann, Transversally perturbed planar dynamical systems . . . . . 187

Theodore Windle Palmer, Real $C^{*}$-algebras ................... 195

Don David Porter, Symplectic bordism, Stiefel-Whitney numbers, and a

Novikov resolution ....................................... 205

Tilak Raj Prabhakar, On a set of polynomials suggested by Laguerre

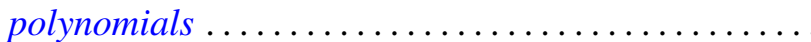

B. L. S. Prakasa Rao, Infinitely divisible characteristic functionals on locally convex topological vector spaces...................

John Robert Reay, Caratheodory theorems in convex product structures ...

Allan M. Sinclair, Eigenvalues in the boundary of the numerical range. .

David R. Stone, Torsion-free and divisible modules over matrix rings ....

William Jennings Wickless, A characterization of the nil radical of a

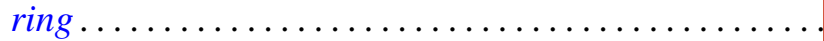

